\title{
Correlations Between X-ray and Radio Pulses in Vela
}

\author{
Jennifer Donovan ${ }^{1}$, Andrea Lommen \\ Franklin and Marshall College, Lancaster, Pennsylvania, USA \\ Zaven Arzoumanian ${ }^{2}$, Alice Harding \\ NASA Goddard Space Flight Center, Greenbelt, Maryland, USA \\ Mark Strickman \\ Code 7651.2, Naval Research Laboratory, Washington, DC, USA \\ Carl Gwinn \\ University of California, Santa Barbara, California, USA \\ Richard Dodson, Peter McCulloch \\ University of Tasmania, Tasmania, Australia \\ David Moffett \\ Furman University, Greenville, South Carolina, USA
}

\begin{abstract}
Pulse-shape changes correlating with flux have been shown to exist in the Vela pulsar's radio emission (Krishnamohan \& Downs 1983). The aim of this work was to look for correlations between radio and X-ray flux in Vela on a pulse-by-pulse basis. We found that Vela emits significantly more X-rays during radio pulses with strong flux than during weak radio flux pulses, which indicates that the mechanisms creating the Vela pulsar's X-ray and radio emission must be related.
\end{abstract}

\section{Procedure}

Using the X-ray and radio emission from the Vela pulsar, we showed that the emission mechanisms at work in these two very different bands must be related. Our data consist of simultaneous radio and X-ray observations at the Mount Pleasant Radio Observatory in Tasmania at a frequency of $985 \mathrm{MHz}$ and the $R X T E$ satellite in the energy range of $2-16 \mathrm{keV}$ (Harding et al. 2002). X-ray photon arrival times were transformed to the Solar System barycenter, and the

\footnotetext{
${ }^{1}$ Currently at Columbia University.

${ }^{2}$ Universities Space Research Association.
} 
radio ephemeris was converted to infinite frequency using the pulsar dispersion measure. Each X-ray photon was then matched with the radio pulse during which it was emitted, and the X-ray photons were separated into one of four intensity bins depending on the associated pulse's radio flux.

Ensuring that scintillation was not a problem was a particularly relevant issue for this project. Diffractive scintillation proved to be unimportant as Vela's diffractive bandwidth at $985 \mathrm{MHz}$ is $2 \mathrm{kHz}$, while our observational bandwidth was $6.4 \mathrm{MHz}$; in effect, we were averaging over approximately 3200 "scintles." Refractive scintillation was also not a concern within any single radio observation, but it did affect the received radio flux from one observation to the next. In order to account for this, we redefined for each individual radio observation the radio flux cutoffs for each flux bin (e.g., the specific radio flux that separated weak pulses from "med-weak" pulses). These cutoffs were defined in such a way that an equal number of radio pulses was associated with each strength bin.

\section{Results}

We found that Vela emits significantly more X-rays during the pulses that were strong in radio wavelengths than during the pulses that were weak in radio wavelengths. We used all X-ray photons emitted during radio pulses for our experiment, and ongoing work will test whether this correlation arises due primarily to certain components of the pulse profile.
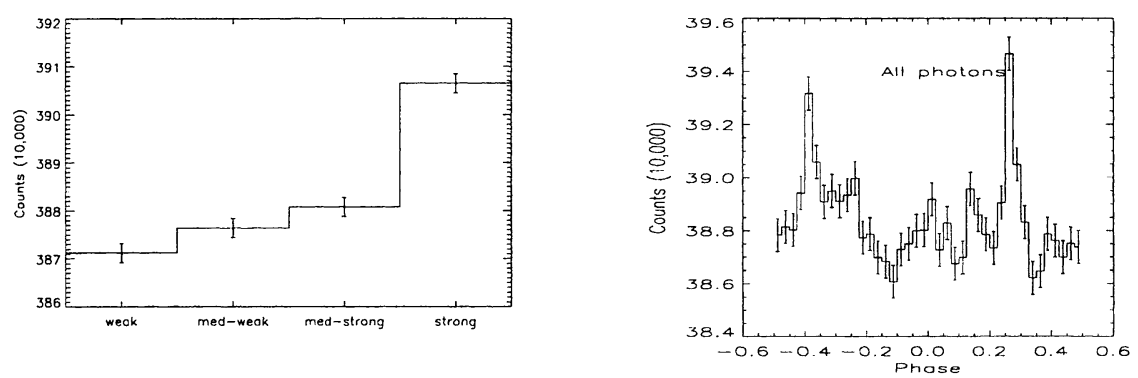

Figure 1. All utilized X-ray data, totaling 264959 seconds. The first plot shows the number of X-ray photons that fell into each radio flux strength bin, and the second shows the full integrated X-ray pulse profile where the radio peak falls at phase 0.15 . Note that the minimum value of the ordinate in the first plot is $3.86 \times 10^{6} \mathrm{X}$-ray counts.

Acknowledgments. Thanks to D. Helfand, E. Praton, the Anton Pannekoek Institute (Amsterdam), Franklin and Marshall, and NSF grant 0107342.

\section{References}

Harding, A. K., Strickman, M. S., Gwin, C., Dodson, R., Moffet, D., \& McCulloch, P. 2002, ApJ, 576, 376

Krishnamohan, S., \& Downs, G. S. 1983, ApJ, 265, 372 